\title{
Avaliação do consumo da merenda escolar em escolas municipais de Porto Alegre*
}

\author{
Evaluation of the consumption of meals supplied in Porto Alegre's plublic schools
}

\section{Alexandre Bulsing Hernández ${ }^{1}$, Sonia Maria Blauth de Slavutzky ${ }^{2}$, Dalva Maria Pereira Padilha ${ }^{3}$}

\section{Resumo}

Os objetivos deste estudo foram descrever os componentes da dieta dos alunos do Segundo e Terceiro Ciclos de quatro Escolas Municipais de Porto Alegre, verificar se a merenda escolar fornecida pela instituição é consumida pelos estudantes e, também, verificar se a existência de cantina nas escolas modifica o consumo desta merenda.

Utilizou-se, de forma aberta um recordatório de vinte e quatro horas que avaliou a dieta de 1398 alunos.

Verificou-se que a merenda escolar fornecida pela instituição é pouco consumida pelos estudantes, uma vez que é disponibilizada a todos e apenas $41,8 \%$ dos participantes do estudo consumiram esta alimentação.

$\mathrm{O}$ consumo de itens externos à merenda institucional, na sua maioria nutricionalmente fracos e com altos teores de açúcar e gorduras, deve ser considerado um fator que colabora com o não consumo da merenda, além de ser um fator de risco para o estabelecimento de doenças crônico-degerenativas. Verificou-se que há comércio de gêneros nas quatro escolas avaliadas, o que impossibilitou a comparação entre as escolas com e sem cantina. Observou-se ainda que o ambiente escolar é pouco aproveitado para o desenvolvimento de atividades de educação alimentar. Sugere-se que o elevado percentual de alunos que não consomem a merenda institucional deveria ser interpretado como um desperdício de recursos públicos.

Palavras-chave: Alimentação escolar, Promoção da saúde, Prevenção de doenças, Nutrição de grupos de risco

\section{Abstract}

The objectives of this observational descriptive and crosssectional study were: 1 ) to describe the diet components of students from the second and third cycles of Porto Alegre public schools, 2) to verify if the institutional school meal offered was eaten by the students and 3) to verify if the presence of canteens inside the schools modified the consumption.

A twenty four hour opened reminder was used to evaluate the diet of 1398 students.

It was observed that the institutional school meal was hardly consumed by the students, since it was offered to all of them and only $41,8 \%$ ate the meal.

The consumption of items external to the school meal, were in the great majority, nutritionally weak, with high quantity of sugar and fat. These items must be considered an important factor for the non-consumption of the school meal, besides being a risk factor for the development of chronic degenerative diseases. It was verified that there was an informal commerce of food items inside the four schools studied, what made impossible the comparison between schools with and without canteens. It was observed that the school environment is hardly used for the development of alimentary education activities. This study suggests that the high percentage of students that do not eat the institutional school meal should be interpreted also a waste of public funds.

Keywords: School feeding, Health promotion, Disease prevention, Risk groups nutrition.
${ }^{1}$ Mestre em Saúde Bucal Coletiva pela Faculdade de Odontologia da Universidade Federal do Rio Grande do Sul. Professor Substituto do Departamento de Odontologia Social e Preventiva da Faculdade de Odontologia da Universidade Federal do Rio Grande do Sul. Responsável pela elaboração do projeto, coleta, análise de dados e redação do artigo.

2 Professora Doutora do Departamento de Odontologia Social e Preventiva da Faculdade de Odontologia da Universidade Federal do Rio Grande do Sul. Pesquisadora principal, responsável pela orientação do projeto, análise de dados e redação do artigo.

3 Professora Doutora do Departamento de Odontologia Social e Preventiva da Faculdade de Odontologia da Universidade Federal do Rio Grande do Sul.

* Baseado na Dissertação de Mestrado intitulada "Avaliação da dieta em Escolas Municipais de Porto Alegre com relação ao consumo de sacarose/2004" (p. 88) apresentada no Programa de Pós-Graduação em Odontologia da Faculdade de Odontologia da Universidade Federal do Rio Grande do Sul, em agosto de 2005, como parte do requisito à obtenção do grau de Mestre em Saúde Coletiva pela FO-UFRGS.

Correspondência - Alexandre Bulsing Hernández

Endereço: Rua Cel. Vicente, 408/1103 - CEP 90030-040 - Porto Alegre - RS.

Fone: 5132398873 ou 5199568873

E-mail: abhz@terra.com.br

\section{Introdução}

Moysés e Watt (2000) afirmam que a construção de políticas públicas saudáveis e a criação de ambientes que apóiem as escolhas saudáveis são requisitos básicos para a promoção de saúde. $O$ Programa Nacional de Alimentação Escolar (PNAE) brasileiro é um dos maiores programas na área de alimentação escolar no mundo, tendo atingido, em 2005, 36,4 milhões de escolares (BRASIL, 2004).

Programas de alimentação escolar são realizados para melhorar a frequência, o aprendizado, o crescimento, entre outros fatores (KRISTJANSSON et al., 2007).

Pobres resultados em saúde como o sobrepeso e a obesidade em crianças têm crescido rapidamente em muitos países (KAUFMAN; KARPATI, 2007; LOBSTEIN; MILLSTONE, 2007). Além disso, de acordo com Abreu, Pordeus e Modena (2004), a cárie dentária ainda representa o principal problema de saúde bucal coletiva no Brasil, e sua prevalência permanece crescendo em paises em desenvolvimento e os fatores de risco relacionados ao consumo de açúcar são comuns a outros problemas de saúde (MOYNIHAN; PETERSEN, 2004).

Cavalcante, Priore e Franceschini (2004) destacam que a avaliação do consumo alimentar tem um papel crítico na área de pesquisa em nutrição e saúde e também no desenvolvimento de programas.

Existem algumas controvérsias sobre a efetividade de programas de alimentação escolar. Enquanto alguns estudos apontam para o beneficio imediato da redução da fome entre os escolares, outros colocam que há poucas evidências desses benefícios que só seriam visíveis quando aliados a outras políticas de melhoria da qualidade escolar. "Há o questionamento que os programas de alimentação escolar são direcionados ao sintoma, sem buscar as principais causas da fome" (MCINTYRE; DAYLE, 1992).

Nos últimos anos, não faltam casos que comprovam a existência de uma situação frágil do Programa da merenda, seja por meio das recorrentes denúncias de desvio do dinheiro destinado à compra de alimentos, seja pela simples constatação da falta de comida nas escolas ao longo de meses ou, ainda, pelo fornecimento de uma merenda escassa e pobre em nutrientes (ASSOCIAÇÃO DE APOIO A POLÍTICAS DE SEGURANÇA ALIMENTAR, 2005). 
Dessa forma, a avaliação de programas de alimentação escolar é de crucial importância para manutenção, fiscalização e acompanhamento desta estratégia. São, portanto, objetivos do presente estudo descrever os principais componentes da dieta de 1398 alunos do Ensino Fundamental de Escolas Municipais de Porto Alegre, região sul do Brasil; verificar se a merenda escolar fornecida pela instituição é consumida pelos estudantes; e, também, verificar se a existência de cantina nas escolas modifica, entre os alunos, o consumo desta merenda.

\section{Materiais e Métodos}

Quatro escolas da Rede Municipal de Ensino, no ano de 2004, foram incluídas neste estudo observacional, do tipo descritivo transversal, no qual o consumo de merenda escolar por 1398 estudantes matriculados no Segundo e Terceiro Ciclos foi avaliado. De acordo com a listagem fornecida pela Secretaria Municipal de Educação de Porto Alegre, Brasil, (SMED), no ano de 2003, existiam quarenta e cinco Escolas Municipais de Ensino Fundamental (alunos a partir de sete anos de idade). Destas foram identificadas cinco escolas que possuíam cantina nas suas dependências. Procedeu-se, então, a localização das escolas com cantina (Escolas 1 e 3 ) nas Regiões do Orçamento Participativo (ROPs) e buscou-se um pareamento de escolas sem cantina de mesmo porte (Escolas 2 e 4) na mesma região. Nas ROPs onde mais de uma escola enquadrou-se nos critérios estabelecidos, procedeu-se um sorteio para definir a escola a ser estudada. A definição do número de escolas que participariam do estudo foi definida segundo o tempo e os recursos disponíveis. Dessa maneira, foram selecionadas duas Escolas na zona sul (Escolas 1 e 2) e duas Escolas na zona norte (Escolas 3 e 4) de Porto Alegre.

A região Sul é composta pelo bairro Restinga e tem 53.764 habitantes, representando $3,95 \%$ da população do município. Com área de $38,56 \mathrm{~km}^{2}$, representa $8,10 \%$ da área do município, sendo sua densidade demográfica de 1.394,29 habitantes por $\mathrm{km}^{2}$. A taxa de analfabetismo é de $6,0 \%$ e o rendimento médio dos responsáveis por domicílio é de 3,6 salários mínimos.

A região Norte é composta pelo bairro Sarandi e tem 90.665 habitantes, representando $6,66 \%$ da população do município. Com área de $28,76 \mathrm{~km}^{2}$, representa $6,04 \%$ da área do município, sendo sua densidade demográfica de $3.152,47$ habitantes por $\mathrm{km}^{2}$. A taxa de analfabetismo é de $4,9 \%$ e o rendimento médio dos responsáveis por domicílio é de 4,4 salários mínimos.

Todos os estudantes que participaram do estudo receberam um formulário de Recordatório de Vinte e Quatro Horas, conforme proposto por Cavalcante, Priore e Franceschini, em 2004, para preenchimento. Em cada escola, foi realizada uma coleta de dados e as quatro escolas foram avaliadas na primeira quinzena do mês de novembro de 2004, sendo que a primeira semana foi reservada para a apresentação do trabalho e solicitação das autorizações e a segunda, à coleta de dados.

Os alunos foram orientados a escrever tudo o que ingeriram, a procedência e as respectivas quantidades, desde o momento em que iniciou o turno escolar até o final desse período. Foram, também, orientados a identificar a ficha com o nome e a idade para, dessa forma, evitar que mais de uma ficha fosse preenchida pelo mesmo aluno e permitir a identificação das autorizações. Foi sugerido aos alunos que, no dia anterior à coleta dos dados, ou seja, no dia avaliado, ao longo do período, escrevessem, em uma folha do caderno, tudo o que estavam ingerindo logo após o consumo, para que a informação fosse bastante completa e precisa.

Os dados foram tabulados e analisados através do programa Epi-Info. Versão 3.3. Além da estatística descritiva, o Teste Qui-Quadrado foi usado com o intuito de verificar se o consumo de itens externos deve ser considerado entre fatores de risco para o não consumo da merenda fornecida pelas escolas.
Obteve-se, para o desenvolvimento do estudo, além do consentimento da SMED, das Diretorias das escolas e dos responsáveis pelos alunos, submissão e aprovação do Comitê de Ética e Pesquisa da Faculdade de Odontologia da UFRGS, que o julgou eticamente adequado, segundo a resolução 196/96 da Comissão Nacional de Ética em Pesquisa, obedecendo assim aos princípios básicos às investigações estabelecidos na VI Declaração de Helsinki.

\section{Resultados}

Entre as quatro escolas, foram obtidos 1659 questionários e desses, 1.398 foram considerados válidos. Os alunos que não receberam a autorização dos pais e os alunos que manifestaram o desejo de não participar do estudo foram orientados a não preencher o questionário. Foram considerados inválidos os questionários em que não foi possível compreender o que estava escrito e os questionários nos quais o aluno não compreendeu a orientação para o preenchimento. Os alunos que não compareceram no dia em que a dieta foi avaliada foram orientados a relatar que estavam ausentes.

Os valores descritos na Tabela 1 representam os valores absolutos e percentuais do total de questionários que relataram ter consumido itens alheios à merenda fornecida pelas escolas, bem como as respectivas procedências. A diferença conceitual entre "Comprou na escola" e "Comprou no bar da escola" é explicável, uma vez que, mesmo nas escolas sem cantina, no seu espaço físico, há pessoas que comercializam salgados, bolos, guloseimas, refrigerantes, biscoitos, entre outros produtos.

Tabela 1. Valores Absolutos e Percentuais do Número de Questionários que Indicaram o Consumo de Itens Externos à Merenda Institucional, Segundo a Procedência do Item. Porto Alegre, RS, 2004.

\begin{tabular}{|c|c|c|c|c|c|c|}
\hline $\begin{array}{l}\text { Item Externo } \\
\text { à Merenda } \\
\text { Institucional }\end{array}$ & $\begin{array}{l}\text { Trouxe } \\
\text { de } \\
\text { Casa }\end{array}$ & $\begin{array}{l}\text { Comprou } \\
\text { na } \\
\text { Escola }\end{array}$ & $\begin{array}{l}\text { Comprou } \\
\text { no Bar da } \\
\text { Escola }\end{array}$ & Outras & $\begin{array}{l}\text { Total de } \\
\text { Questionários }\end{array}$ & $\%$ \\
\hline Balas $\left(1.413^{\star}\right)$ & 21 & 70 & 144 & 123 & 358 & 25,6 \\
\hline $\begin{array}{l}\text { Chicletes } \\
\left(265^{\star}\right)\end{array}$ & 12 & 37 & 13 & 66 & 128 & 9,2 \\
\hline Pirulitos $\left(299^{\star}\right)$ & 12 & 76 & 9 & 47 & 144 & 10,3 \\
\hline $\begin{array}{l}\text { Outras Gulo- } \\
\text { seimas }\end{array}$ & 8 & 5 & 27 & 14 & 54 & 3,9 \\
\hline $\begin{array}{l}\text { Biscoitos } \\
\text { Doces }\end{array}$ & 43 & 30 & 7 & 98 & 178 & 12,7 \\
\hline Bolos e Afins & 8 & 36 & 23 & 11 & 78 & 5,6 \\
\hline $\begin{array}{l}\text { Biscoitos } \\
\text { Salgados }\end{array}$ & 12 & 0 & 0 & 7 & 19 & 1,4 \\
\hline Salgadinhos & 13 & 12 & 30 & 50 & 105 & 7,5 \\
\hline $\begin{array}{l}\text { Salgados e } \\
\text { Afins }\end{array}$ & 14 & 49 & 63 & 18 & 144 & 10,3 \\
\hline Frutas & 5 & 0 & 1 & 2 & 8 & 0,6 \\
\hline Refrigerantes & 35 & 22 & 49 & 21 & 127 & 9,1 \\
\hline
\end{tabular}

e Sucos

Nota: Os valores percentuais referidos têm como base os 1.398 questionários válidos. * Número de unidades consumidas.

É possível verificar que o item Balas foi relatado o maior número de vezes, sendo citado por mais de um quarto dos alunos que participaram do estudo. Verificou-se que, entre os alunos que consumiram balas, cada um consumiu, em média, 3,95 balas durante um turno escolar. Quando não fornecida pela merendar escolar, apenas oito $(0,6 \%)$ dos 1398 alunos, relataram ter consumido algum tipo de fruta.

A merenda institucional fornecida pelas escolas é oferecida na forma de bufê, o que pode causar uma variação entre o tamanho 
das porções servidas, pois cada aluno serve sua própria porção. Assim, ao referir acerca do consumo, tanto para o lanche quanto para a refeição que é fornecida pelas instituições, considerou-se como Consumo Completo quando todas as variedades oferecidas tiveram seus consumos citados pelos alunos; Consumo Parcial quando o aluno relatou ter consumido a merenda institucional, mas não foram consumidas todas as variedades oferecidas; Sem Consumo quando nenhum dos itens consumidos no ambiente escolar procedeu do refeitório da escola. A Tabela 2 apresenta a frequência e o tipo de consumo de lanche e de refeição oferecidos em cada uma das escolas participantes do estudo.

Tabela 2. Frequência e tipo de consumo de Lanche e de Refeição oferecidos pelas escolas, Porto Alegre, RS, 2004.

\begin{tabular}{|c|c|c|c|c|c|c|c|c|c|}
\hline \multirow{3}{*}{ Escola } & \multicolumn{9}{|c|}{ Consumo de Refeição da Instituição } \\
\hline & \multirow{2}{*}{$\begin{array}{l}\text { Consumo } \\
\text { de } \\
\text { Lanche }\end{array}$} & \multicolumn{2}{|c|}{ Completo } & \multicolumn{2}{|c|}{ Parcial } & \multicolumn{2}{|c|}{$\begin{array}{c}\text { Sem } \\
\text { Consumo }\end{array}$} & \multicolumn{2}{|c|}{ Total } \\
\hline & & $\mathrm{N}$ & $\%$ & $\mathrm{~N}$ & $\%$ & $\mathrm{~N}$ & $\%$ & $\mathrm{~N}$ & $\%$ \\
\hline \multirow{3}{*}{$\begin{array}{l}1 \\
(n=398)\end{array}$} & Completo & 6 & 1,5 & 7 & 1,8 & 10 & 2,5 & 23 & 5,8 \\
\hline & Parcial & 3 & 0,8 & 10 & 2,5 & 26 & 6,5 & 39 & 9,8 \\
\hline & $\begin{array}{l}\text { Sem } \\
\text { Consumo }\end{array}$ & 11 & 2,7 & 68 & 17,1 & 257 & 64,6 & 336 & 84,4 \\
\hline \multirow{5}{*}{$\begin{array}{l}2 \\
(n=378)\end{array}$} & Total & 20 & 5,0 & 85 & 21,4 & 293 & 73,6 & 398 & 100 \\
\hline & Completo & 10 & 2,6 & 21 & 5,6 & 25 & 6,6 & 56 & 14,8 \\
\hline & Parcial & 3 & 0,8 & 7 & 1,9 & 25 & 6,6 & 35 & 9,3 \\
\hline & $\begin{array}{l}\text { Sem } \\
\text { Consumo }\end{array}$ & 17 & 4,5 & 34 & 9,0 & 236 & 62,4 & 287 & 75,9 \\
\hline & Total & 30 & 7,9 & 62 & 16,4 & 286 & 75,7 & 378 & 100 \\
\hline \multirow{3}{*}{$\begin{array}{l}3 \\
(n=166)\end{array}$} & Completo & 6 & 3,6 & 16 & 9,6 & 11 & 6,6 & 33 & 198 \\
\hline & Parcial & 2 & 1,2 & 7 & 4,2 & 15 & 9,0 & 24 & 144 \\
\hline & $\begin{array}{l}\text { Sem } \\
\text { Consumo }\end{array}$ & 22 & 13,3 & 28 & 16,9 & 59 & 35,5 & 109 & 65,7 \\
\hline \multirow{5}{*}{$\begin{array}{l}4 \\
(n=456)\end{array}$} & Total & 30 & 18,1 & 51 & 30,7 & 85 & 51,2 & 166 & 100 \\
\hline & Completo & 14 & 3,1 & 8 & 1,8 & 37 & 8,1 & 59 & 13,0 \\
\hline & Parcial & 6 & 1,3 & 5 & 1,1 & 50 & 11,0 & 61 & 13,4 \\
\hline & $\begin{array}{l}\text { Sem } \\
\text { Consumo }\end{array}$ & 32 & 7,0 & 43 & 9,4 & 261 & 57,2 & 336 & 73,6 \\
\hline & Total & 52 & 11,4 & 56 & 12,3 & 348 & 76,3 & 456 & 100 \\
\hline \multirow{4}{*}{$\begin{array}{l}\text { Total } \\
(n= \\
1398)\end{array}$} & Completo & 36 & 2,6 & 52 & 3,7 & 83 & 5,9 & 171 & 12,2 \\
\hline & Parcial & 14 & 1,0 & 29 & 2,1 & 116 & 8,3 & 159 & 11,4 \\
\hline & $\begin{array}{l}\text { Sem } \\
\text { Consumo }\end{array}$ & 82 & 5,8 & 173 & 12,4 & 813 & 58,2 & 1068 & 76,4 \\
\hline & Total & 132 & 9,4 & 254 & 18,2 & 1012 & 72,4 & 1398 & 100 \\
\hline
\end{tabular}

Nota: Os valores representados são referentes ao total de questionários válidos obtidos em cada escola.

É possível verificar que o Consumo Completo do lanche e da refeição institucional foi de 2,6\%, sendo maior na Escola 3 (3,6\%) e menor na Escola 1 (1,5\%). Entre as quatro escolas, $58,2 \%$ dos alunos não fizeram consumo nem do lanche nem da refeição oferecida pela instituição e, individualmente, esse percentual foi maior na Escola 1 (64,6\%) e menor na Escola 3 (35,5\%).

A comparação dos itens apresentados na Tabela 1 e na Tabela 2 é apresentada na Tabela 3, que considera a dieta dos estudantes no ambiente escolar, tanto dos alimentos fornecidos pela instituição, quanto dos itens alheios ao refeitório escolar. Observa-se que $23,2 \%$ dos estudantes afirmaram ter consumido apenas alimentos fornecidos pela escola, 18,6\% consumiram tanto os itens oferecidos pela escola quanto os externos a ela, $33 \%$ ingeriram apenas produtos alheios à merenda institucional e o não consumo de nenhum dos itens foi relatado por $25,2 \%$ dos alunos.
Tabela 3. Relação entre o consumo de itens externos em relação ao consumo da Alimentação Escolar nas Escolas Municipais de Porto Alegre, RS, 2004.

\begin{tabular}{|c|c|c|c|c|c|c|c|}
\hline & & \multicolumn{4}{|c|}{$\begin{array}{l}\text { Consumo da Alimentação } \\
\text { Escolar }\end{array}$} & \multirow{3}{*}{$\begin{array}{l}\text { OR IC } \\
(95 \%)\end{array}$} & \multirow{3}{*}{$p$} \\
\hline & & \multicolumn{2}{|c|}{ Não } & \multicolumn{2}{|c|}{ Sim } & & \\
\hline & & $\mathrm{N}$ & $\%$ & $\mathrm{~N}$ & $\%$ & & \\
\hline \multirow{2}{*}{$\begin{array}{l}\text { Consumo } \\
\text { de Itens } \\
\text { Externos }\end{array}$} & Sim & 461 & 33,0 & 260 & 18,6 & \multirow{2}{*}{$\begin{array}{c}1,64 \\
{[1,31 ;} \\
2,04]\end{array}$} & \multirow[t]{2}{*}{$<0,01$} \\
\hline & Não & 352 & 25,2 & 325 & 23,2 & & \\
\hline
\end{tabular}

\section{Discussão}

Neste estudo buscou-se avaliar o consumo de merenda escolar entre estudantes de escolas municipais de Porto Alegre. A análise de 1398 recordatórios de 24 horas preenchidos por esses estudantes demonstrou que a maioria $(58,2 \%)$ desses não consome a merenda da escola e que esta é principalmente substituída por produtos que contêm, na sua composição, o açúcar.

O Programa Nacional de Alimentação Escolar (PNAE) brasileiro está entre os maiores programas de fornecimento de alimentação do mundo, sendo, atualmente, responsável pela alimentação de mais de $20 \%$ da população do país. A prefeitura municipal de Porto Alegre é responsável pela organização da merenda escolar na sua rede, que inclui escolas de áreas desfavorecidas economicamente. O Fundo Nacional para Desenvolvimento da Educação (BRASIL, 2004) destaca que o objetivo do PNAE é atender às necessidades nutricionais dos alunos durante sua permanência em sala de aula, contribuindo para o crescimento, o desenvolvimento, a aprendizagem e o rendimento escolar dos estudantes, bem como a formação de hábitos alimentares saudáveis.

Considerando que onde há maiores níveis de pobreza e maiores desigualdades há mais doença (STARFIELD, 2002), em um país com tantas carências e desigualdades como o Brasil, não é possível convir que $58,2 \%$ das crianças avaliadas no estudo deixem de consumir uma alimentação de qualidade oferecida gratuitamente. O alto consumo de produtos com elevados teores de açúcar observado no presente estudo está de acordo com o fenômeno denominado transição nutricional que vem modificando a dieta do Brasil (MONTEIRO; MONDINI; COSTA, 2000) e de muitos paises, reduzindo o consumo de frutas e vegetais e aumentando o consumo de refrigerantes e produtos ricos em açúcar, levando a um aumento na prevalência de obesidade e de sobrepeso, inclusive em países que, historicamente, apresentavam uma dieta considerada como um modelo de alimentação saudável (LOBSTEIN; MILLSTONE, 2007). A observação da Tabela 3 permite supor que o consumo de itens externos à merenda fornecida pela instituição, quando se considera a dieta na sua totalidade, deve ser visto como uma influência negativa para o consumo da Alimentação Escolar.

A formação de hábitos alimentares saudáveis pode ser considerada uma das funções mais importantes da alimentação escolar. Neste sentido, o relatório sobre Dieta, Nutrição e a Prevenção de Doenças Crônicas (WORLD HEALTH ORGANIZATION, 2000), a Organização Mundial de Saúde (OMS) recomenda que o máximo de calorias provenientes do açúcar esteja entre 6 e 10\% da ingestão diária, o que equivale à ingestão de 40 a 55 gramas pessoa/dia como uma forma de reduzir o risco do desenvolvimento de doenças crônicas.

Com relação ao período da coleta de dados, dado o atual contexto socioeconômico brasileiro, quando o aporte familiar de recursos, em muitas situações, não cobre a totalidade do mês, o período em que os escolares e sua dieta foram avaliados poderia representar um viés. Optou-se, então, por coletar os dados nas quatro escolas no transcorrer da mesma semana. Imagina-se que a decisão mais adequada para não influenciar nas respostas obtidas, trazendo maior fidelidade ao mecanismo de coleta de dados tenha sido a de classificar os itens no momento da tabulação. 
A decisão de utilizar um Recordatório de Vinte e Quatro Horas de forma aberta trouxe a obrigatoriedade de que todos os participantes fossem alfabetizados e, por esse motivo, foram previamente excluídos os alunos das séries iniciais.

A ideia de comparar escolas com e sem estabelecimentos que comercializam produtos alimentares nas suas dependências, não pôde ser desenvolvida, uma vez que há comércio de gêneros tanto formal quanto informal nas escolas e nos seus arredores.

Ao observar a Tabela 2, considerando-se que as quatro escolas oferecem o mesmo padrão de alimentação aos alunos, é possível concluir que as variações na procura pela merenda escolar não podem ser explicadas apenas pelo consumo de itens externos, ainda que estes possivelmente exerçam influência, conforme se observa na Tabela 3. Há uma dinâmica de fatores que interferem no hábito alimentar e que poderiam ser modificados pela aplicação de estratégias da Promoção de Saúde.

Os resultados aqui apresentados apontaram para uma baixa procura pela alimentação oferecida pela escola, demonstrada na Tabela 2, aliada ao consumo exagerado de produtos açucarados e de nutricionalmente pobres, observados na Tabela 1, o que, além de representar o desperdício de recursos públicos, constitui um risco para a saúde dos alunos e comprova a necessidade de utilizar o espaço escolar para promover a educação alimentar.

Nesse sentido, o presente estudo concorda com a conclusão de Costa, Ribeiro e Ribeiro (2001), que apresentam a escola como um espaço e um tempo privilegiados para promover a saúde, por ser um local onde muitas pessoas passam grande parte do seu tempo, vivem, aprendem e trabalham.

Silva e Sturion (2000) salientam que os administradores do PNAE, através da municipalização, têm possibilidades de formular diretrizes nutricionais que levem em consideração o perfil nutricional do público-alvo, e permitindo, dessa forma, desenvolver ações que visem à redução das desigualdades.

"Problemas ambientais, como a ausência de alimentos saudáveis na dieta, são considerados como problemas de estilo de vida pessoal, resolvidos por educação para a saúde". E mais, a explicação das doenças não leva em conta "a interação dinâmica entre os fatores socio-psicológicos" (MOYSÉS; WATT, 2000). É, portanto, necessário que haja um engajamento de toda a comunidade escolar no sentido de promover a saúde dos alunos, sob pena de formar precocemente uma geração de portadores de doenças cronicodegenerativas.

A necessidade de realizar o estudo ora apresentado surgiu de observações feitas pelos autores em atividades educativas desenvolvidas em escolas por uma disciplina da Faculdade de Odontologia da UFRGS, e da discussão que vem se estabelecendo em diversas cidades e estados sobre o estabelecimento de legislação como forma de melhorar a alimentação dos jovens nas escolas.

Há alguns anos, é possível observar o empenho dos setores de nutrição em melhorar a qualidade da merenda oferecida aos estudantes. No entanto, durante as atividades desenvolvidas nas escolas, apesar do valor nutritivo, do odor agradável, da boa aparência e do sabor, é possível constatar que não é uma unanimidade a procura pela alimentação fornecida pela escola. Por outro lado, é amplo o consumo de produtos industrializados, na sua maioria, ricos em aditivos e açúcar e de baixo valor nutricional.

Somando-se a esta constatação, a mídia tem noticiado uma série de iniciativas que buscam a mudança de hábitos alimentares dos estudantes, através da normatização do que é ou não permitido comercializar e consumir no ambiente escolar. A iniciativa, apesar de bem intencionada, se isolada, pode ser de pouca utilidade, pois, sabidamente, apenas pela legislação não é possível obter uma mudança de atitudes. Assim, Freire (1995) afirma que alterações importantes na dieta podem ser obtidas através de abordagens populacionais que utilizem organizações comunitárias, mídia, autoridades e instruções individuais.
A análise da procedência dos itens apresentados na Tabela 1 permite perceber que a solução para a questão da dieta dos escolares precisa ir muito além da legislação sobre as cantinas, uma vez que este estabelecimento é responsável por um baixo percentual dos itens consumidos.

Considerando que as escolas fornecem alimentação aos estudantes, não se justifica a existência de comércio de gêneros no ambiente escolar. Cabe destacar, entretanto, o fato que, entre as quatro escolas avaliadas, a única que apresentava algum tipo de norma sobre os produtos consumidos e comercializados era a Escola 1, onde, por determinação da Diretora e com a anuência dos pais, não era permitida a comercialização de chicletes e pirulitos na cantina, sendo, portanto, o consumo desses itens significativamente menor nessa escola.

Os problemas nutricionais vão desde a mídia até os serviços de saúde. Sendo uma das funções do Programa Nacional de Merenda Escolar a formação de hábitos saudáveis, o consumo da merenda escolar deve ser amplamente estimulado e, devido aos inúmeros malefícios que acarreta o consumo de guloseimas no ambiente escolar, principalmente na sala de aula, deve ser interpretado como uma influência negativa à educação alimentar e à formação de hábitos salutares.

A merenda escolar fornecida pela instituição é pouco consumida pelos estudantes, uma vez que é disponibilizada a todos e apenas $41,8 \%$ dos participantes do estudo consumiram esta alimentação. $\mathrm{O}$ consumo de itens externos à merenda fornecida pela instituição deve ser considerado um fator que colabora com o não consumo da merenda escolar. Dessa forma, observou-se que o ambiente escolar é pouco aproveitado para o desenvolvimento de atividades de educação alimentar. Além disso, sendo a merenda escolar disponibilizada a todos os alunos, o fato de não ser consumida por um percentual considerável desses deveria ser interpretado como mau uso de recursos públicos.

Isoladamente, a existência de cantina nas escolas não pôde ser avaliada, pois verificou-se que há comércio de gêneros nas quatro escolas estudadas. Ao discutir o problema do comércio informal no entorno, ou mesmo dentro das escolas, ouve-se que esta é uma questão complexa, por se tratar de um problema social, deixando a nítida impressão de que o termo social é usado como forma de tornar o problema abstrato, de solução impensável e, principalmente, inatingível, imutável; perpetuando e legitimando, assim, uma atividade baseada no descumprimento das normatizações, como a sonegação fiscal e a falta de vigilância sanitária dos alimentos.

Ainda que este estudo tenha tido várias importantes limitações, pois não foi possível buscar uma amostra representativa do município e foi realizada apenas uma coleta de dados em cada escola quando eventualmente no dia pesquisado a ingestão de alimento estivesse modificada, o que poderia constituir um importante viés, os dados apresentados neste estudo compõem o primeiro e único estudo disponível sobre o efetivo consumo de merenda escolar no Brasil. Os dados da pesquisa, apresentados ao Setor de Nutrição da Secretaria Municipal de Educação evidenciaram a importância do problema, visto que a baixa procura pela alimentação oferecida pela escola aliada ao consumo exagerado de produtos açucarados e nutricionalmente pobres, além de representar o desperdício de recursos públicos, constitui um risco para a saúde dos alunos. 


\section{Referências}

ABREU, M. H. N. G.; PORDEUS, I. A.; MODENA, C. M. Cárie Dentária entre Escolares do Meio Rural de Itaúna (MG), Brasil. Rev. Panam. Salud Publica, Washington, v. 16, n. 5, p. 334-344, Nov. 2004.

ASSOCIAÇÃO DE APOIO A POLÍTICAS DE SEGURANÇA ALIMENTAR - APOIO FOME ZERO. Manual de Gestão Eficiente da Merenda Escolar. 2. ed. São Paulo, p. 82, 2005.

BRASIL. Ministério da Educação. Fundo Nacional de Desenvolvimento da Educação. Programa Nacional de Alimentação Escolar. Alimentação Escolar. Disponível em <http://www.fnde.gov.br/ home/index.jsp?arquivo=/alimentacao_escolar/alimentacao_esc. html>. Acesso em 20 de maio 2004.

CAVAlCANTE, A. A. M.; PRIORE, S. E.; FRANCESCHINI, S. C. C. Estudos de Consumo Alimentar: Aspectos Metodológicos Gerais e o seu Emprego na Avaliação de Crianças e Adolescentes. Rev. Bras. Saúde Matern. Infant., Recife, v. 4, n. 3, p. 229-240, jul./set. 2004.

COSTA, E. Q.; RIBEIRO, V. M. B.; RIBEIRO, E. C. O. Programa de Alimentação Escolar: Espaço de Aprendizagem e Produção de Conhecimento. Rev. Nutr., Campinas, v. 14, n. 3, p. 225-229, set./dez. 2001.

FREIRE, M. C. M. Fatores que Influenciam Hábitos Dietéticos em Relação ao Consumo de Açúcar. Rev. Odontoped., São Paulo, v. 2, n. 4, p. 75-83, abr./jun. 1995.

KAUFMAN, L.; KARPATI, A. Understanding the Sociocultural Roots of Childhood Obesity: Food Practices among Latino Families of Bushwick, Brooklyn. Soc. Sci. Med., Oxford, v. 64, no.11, p. 2177-2188, June 2007.

KRISTJANSSON, E. A. et al. School Feeding for Improving the Physical and Psychosocial Health of Disadvantaged Elementary School Children. Cochrane Database Syst. Rev., Oxford, n. 1, 2007.

LOBSTEIN, T.; MILLSTONE, E. Context for the PorGrow Study: Europe's Obesity Crisis. Obes. Rev., Oxford, v. 8, p. 7-16, May 2007. [Suppl. 2].

MCINTYRE, L.; DAYLE, J. B. Exploratory Analysis of Children's Nutrition Programs in Canada. Soc. Sci. Med., Oxford, v. 35, n. 9, p. 1123-1129, Nov. 1992.

MONTEIRO, C. A.; MONDINI, L.; COSTA, R. B. L. Mudanças na Composição e Adequação Nutricional da Dieta Familiar nas Áreas Metropolitanas do Brasil (1988-1996). Rev. Saúde Pública, São Paulo, v. 34, n. 3, p. 251-258, jun. 2000.

MOYNIHAN, P.; PETERSEN, P. E. Diet, Nutrition and the Prevention of Dental Diseases. Public Health Nutr., Wallingford, UK, v. 7, n. 1A, p. 201-226, Feb. 2004.

MOYSÉS, S. T.; WATT, R. Promoção de Saúde Bucal: Definições. In: BUISCHI, Y. P. Promoção de Saúde Bucal na Clínica Odontológica. São Paulo: Artes Médicas, Cap. 1, p. 1-22, 2000.

SILVA, M. V.; STURION, G. L. O Programa de Alimentação Escolar no Brasil. Bol. SBCTA, Campinas, v. 34, n. 1, p. 1-11, jan./jun., 2000.

STARFIELD, B. Atenção Primária, Equilíbrio entre Necessidades de Saúde, Serviços e Tecnologia. Brasília: UNESCO: Ministério da Saúde, p. 19-43, 2002.

WORLD HEALTH ORGANIZATION. Diet, nutrition and prevention of chronic diseases. Genebra, p. 149, 2003.

Rev. Fac. Odontol. Porto Alegre., Porto Alegre, v. 49, n. 1, p. 26-30, jan./abr., 2008. 